\title{
EXISTENCE OF SOLUTIONS TO SOBOLEV-TYPE PARTIAL NEUTRAL DIFFERENTIAL EQUATIONS
}

\author{
SHRUTI AGARWAL AND DHIRENDRA BAHUGUNA
}

Received 3 January 2005; Revised 30 March 2005; Accepted 31 March 2005

This work is concerned with a nonlocal partial neutral differential equation of Sobolev type. Specifically, existence of the solutions to the abstract formulations of such type of problems in a Banach space is established. The results are obtained by using Schauder's fixed point theorem. Finally, an example is provided to illustrate the applications of the abstract results.

Copyright (C) 2006 S. Agarwal and D. Bahuguna. This is an open access article distributed under the Creative Commons Attribution License, which permits unrestricted use, distribution, and reproduction in any medium, provided the original work is properly cited.

\section{Introduction}

Let $X$ and $Y$ be two real Banach spaces. We consider the following neutral differential equation of Sobolev type with a nonlocal history condition:

$$
\begin{gathered}
\frac{d}{d t}\left[B u(t)+f\left(t, u\left(t-\tau_{1}\right)\right)\right]+A u(t)=g\left(t, u(t), u\left(t-\tau_{2}\right)\right), \quad 0<t \leq T, \\
h\left(\left.u\right|_{[-\tau, 0]}\right)=\phi,
\end{gathered}
$$

where $\tau=\max \left\{\tau_{1}, \tau_{2}\right\}, \tau_{i}>0, i=1,2, T<\infty, \phi \in \mathscr{C}_{0}:=C([-\tau, 0], X)$, and $B$ and $A$ are linear operators with the domains contained in $X$ and the ranges contained in $Y . f$ : $[0, T] \times X \rightarrow Y, g:[0, T] \times X \times X \rightarrow Y$ are two given functions, and the map $h$ is defined from $\mathscr{C}_{0}$ into $\mathscr{C}_{0}$. Here $\mathscr{C}_{t}:=C([-\tau, t] ; X)$ for $t \in[0, T]$ is a Banach space of all continuous functions from $[-\tau, t]$ into $X$ endowed with the norm

$$
\|\psi\|_{t}:=\sup \left\{\|\psi(\theta)\|_{X}: \theta \in[-\tau, t]\right\}
$$

The study of differential equations with nonlocal conditions is of significance due to its applications in problems in physics and other areas of applied mathematics. Byszewski [6] proved the existence of mild, strong, and classical solutions for the nonlocal Cauchy problem. Some more results on the existence, uniqueness, and stability of solutions are 
given by Byszewski and Akca [7], Balachandran and Chandrasekaran [2], and Byszewski and Lakshmikantham [8]. Recently, Bahuguna [1] obtained existence and regularity results for functional differential equation with nonlocal condition using semigroup theory. Brill [5] and Showalter [14, 15] established the existence of solutions of semilinear evolution equations of Sobolev type in Banach spaces. On the other hand, Balachandran and Uchiyama [4] considered an integrodifferential equation of Sobolev type with a nonlocal condition and proved the existence of mild and strong solutions.

The theory of neutral differential equations has been extensively studied in the literature. Hernández [10] established the existence results for partial neutral functional differential equations with nonlocal conditions modeled as

$$
\begin{gathered}
\frac{d}{d t}\left(u(t)+F\left(t, u_{t}\right)\right)=A u(t)+G\left(t, u_{t}\right), \quad 0 \leq t \leq T, \\
u_{\sigma}=\phi+q\left(x_{t_{1}}, x_{t_{2}}, \ldots, x_{t_{n}}\right) \in \Omega,
\end{gathered}
$$

where $A$ is the infinitesimal generator of an analytic semigroup $T(t)$ on a Banach space. He made use of fixed point theorems and the results mentioned in Pazy's [13]. For results on neutral partial differential equations with nonlocal and classical conditions, we refer to the papers of Hernández and Henríquez [11], Balachandran and Sakthivel [3], Fu and Ezzinbi [9], and references therein.

Our aim in this paper is to study the existence of a solution of partial neutral differential equation of form (1.1) by using Schauder's fixed point theorem. For this purpose, we first prove the existence of a solution of $(1.1)$ on $[-\tau, \widetilde{T}]$ for some $0<\widetilde{T} \leq T$ and then prove that this solution can be extended to a solution of $(1.1)$ either on $[-\tau, T]$ or on the maximal interval of existence $\left[-\tau, t_{\max }\right], 0<t_{\max } \leq T$, and in the latter case we show that $\lim _{t \rightarrow t_{\max }}\|u(t)\|_{X}=\infty$. Finally, we present an example to show an application of the existence theorem.

\section{Preliminaries}

In this section, we introduce notations, definitions, and preliminary results which we require to establish the existence of a solution of (1.1).

Henceforth, $\left(X,\|\cdot\|_{X}\right)$ and $\left(Y,\|\cdot\|_{Y}\right)$ will denote two real Banach spaces. The space of continuous linear mapping from $X$ into $Y$ will be denoted by $L(X, Y)$.

To prove our main theorem, we consider the following assumptions on the operators $A: D(A) \subset X \rightarrow Y$ and $B: D(B) \subset X \rightarrow Y:$

(i) $A$ and $B$ are closed, linear operators,

(ii) $D(B) \subset D(A)$ and $B$ is bijective,

(iii) $B^{-1}: Y \rightarrow D(B)$ is compact.

From the above fact and the closed graph theorem, we get the boundedness of the linear operator $A B^{-1}: Y \rightarrow Y$. Consequently, $A B^{-1}$ generates a uniformly continuous semigroup $e^{-t A B^{-1}}, t \geq 0$.

Further, we assume that

(iv) there exists a Lipschitz continuous function $\chi \in \mathscr{C}_{0}$ such that $h(\chi)=\phi$ on $[-\tau, 0]$ and $\chi(0) \in D(B)$, 
(v) $f:[0, T] \times X \rightarrow Y$ is a Lipschitz continuous function, that is,

$$
\|f(t, u)-f(s, v)\|_{Y} \leq L\left[|t-s|+\|u-v\|_{X}\right]
$$

for all $t \in[0, T]$ and $u, v \in X . L>0$ is a Lipschitz constant.

Next, we choose a $0<\widetilde{T} \leq T$ such that $\widetilde{T}=\min \left\{\tau_{1}, \tau_{2}\right\}$, then for $0<t \leq \widetilde{T}$, the functions $u\left(t-\tau_{1}\right)$ and $u\left(t-\tau_{2}\right)$ transform to known function $\chi$.

Under the above assumptions, we prove the existence of a solution $u$ of (1.1) in the sense that there exists a continuous function $u:[-\tau, \widetilde{T}] \rightarrow X$ such that $u(t) \in D(B)$ for all $t \in[0, \tilde{T}]$, and in addition, $\left[B u(t)+f\left(t, \chi\left(t-\tau_{1}\right)\right)\right]$ is differentiable on $(0, \tilde{T}]$ satisfying

$$
\begin{gathered}
\frac{d}{d t}\left[B u(t)+f\left(t, \chi\left(t-\tau_{1}\right)\right)\right]+A u(t)=g\left(t, u(t), \chi\left(t-\tau_{2}\right)\right), \quad 0<t \leq \widetilde{T}, \\
u=\chi, \quad \text { on }[-\tau, 0] .
\end{gathered}
$$

Note that $B u(t)$ itself may not be differentiable on the interval of existence.

Now we mention a few results needed to establish our main result.

Proposition 2.1. Suppose the assumptions (i)-(v) are satisfied. Let $F:[0, T] \rightarrow Y$ and $\widetilde{F}:[0, T] \rightarrow Y$ be Bochner integrable functions and let

$$
\begin{aligned}
U(t) & =B u(t)+H(t), \\
\tilde{U}(t) & =B \tilde{u}(t)+\tilde{H}(t), \\
w & =\left\|A B^{-1}\right\|_{Y} .
\end{aligned}
$$

If $u, \tilde{u}$ are solutions of

$$
\begin{gathered}
\frac{d}{d t}[B u(t)+H(t)]+A u(t)=F(t), \\
u=\chi, \quad \text { on }[-\tau, 0] ; \\
\frac{d}{d t}[B \tilde{u}(t)+\tilde{H}(t)]+A \tilde{u}(t)=\tilde{F}(t), \\
\tilde{u}=\tilde{\chi}, \quad \text { on }[-\tau, 0],
\end{gathered}
$$

respectively, where

$$
H(t)=f\left(t, \chi\left(t-\tau_{1}\right)\right), \quad \tilde{H}(t)=f\left(t, \tilde{\chi}\left(t-\tau_{1}\right)\right), \quad \forall[0, T] .
$$

Then, the following hold for $0 \leq s \leq t \leq T$ :

(I)

$$
\begin{aligned}
\|U(t)-\tilde{U}(t)\|_{Y} \leq & e^{w(t-s)}\|U(s)-\tilde{U}(s)\|_{Y}+\int_{s}^{t} e^{w(t-\eta)}\|F(\eta)-\tilde{F}(\eta)\|_{Y} d \eta \\
& +\int_{s}^{t} e^{w(t-\eta)} w\|H(\eta)-\tilde{H}(\eta)\|_{Y} d \eta,
\end{aligned}
$$


4 Sobolev-type neutral partial differential equations

(II)

$$
\|U(t)-U(s)\|_{Y} \leq \int_{s}^{t} e^{w(t-\eta)}\|F(\eta)-A u(s)\|_{Y} d \eta+\int_{s}^{t} e^{w(t-\eta)} w\|H(\eta)-H(s)\|_{Y} d \eta,
$$

(III)

$$
\begin{aligned}
\|A u(t)-A \chi(0)\|_{Y} \leq w & {\left[\int_{0}^{t} e^{w(t-\eta)}\|F(\eta)-A \chi(0)\|_{Y} d \eta\right.} \\
& \left.+\int_{0}^{t} e^{w(t-\eta)} w\|H(\eta)-H(0)\|_{Y} d \eta+\|H(t)-H(0)\|_{Y}\right] .
\end{aligned}
$$

Proof. Let $Y^{*}$ be the dual space of $Y$ and let the duality map $\mathscr{\mathscr { S }}: Y \rightarrow 2^{Y^{*}}$ be given by

$$
\mathscr{J}(y)=\left\{y^{*} \in Y^{*}:\left\langle y, y^{*}\right\rangle=\|y\|_{Y}^{2}=\left\|y^{*}\right\|_{Y^{*}}^{2}\right\} .
$$

Here, $2^{Y^{*}}$ denotes the power set of $Y^{*},\|\cdot\|_{Y}$ and $\|\cdot\|_{Y^{*}}$ are the norms of $Y$ and $Y^{*}$ respectively, and $\left\langle y, y^{*}\right\rangle$ is the value of $y^{*} \in Y^{*}$ at $y \in Y$.

Define a function $\langle\cdot, \cdot\rangle: Y \times Y \rightarrow \mathbb{R}$ by

$$
\langle x, y\rangle:=\inf \left\{\left(x, y^{*}\right): y^{*} \in F(y)\right\} .
$$

(I) Since $\|U-\tilde{U}\|_{Y}:[0, T] \rightarrow \mathbb{R}$ is a differentiable function for a.e. $\eta \in(0, T)$, we may apply the result of Kato [12, Lemma 1.3]. Thus, we have

$$
\begin{aligned}
\| U(\eta) & -\tilde{U}(\eta)\left\|_{Y}\left(\frac{d}{d \eta}\right)\right\| U(\eta)-\tilde{U}(\eta) \|_{Y} \\
& =\langle F(\eta)-A u(\eta)-\tilde{F}(\eta)+A \tilde{u}(\eta), U(\eta)-\tilde{U}(\eta)\rangle \\
& \leq\|F(\eta)-\tilde{F}(\eta)\|_{Y}\|U(\eta)-\tilde{U}(\eta)\|_{Y}+\|A u(\eta)-A \tilde{u}(\eta)\|_{Y}\|U(\eta)-\tilde{U}(\eta)\|_{Y} .
\end{aligned}
$$

It implies

$$
\begin{aligned}
\frac{d}{d \eta}\|U(\eta)-\tilde{U}(\eta)\|_{Y} & \leq\|F(\eta)-\tilde{F}(\eta)\|_{Y}+w\|B u(\eta)-B \tilde{u}(\eta)\|_{Y} \\
& \leq\|F(\eta)-\tilde{F}(\eta)\|_{Y}+w\left[\|U(\eta)-\tilde{U}(\eta)\|_{Y}+\|H(\eta)-\tilde{H}(\eta)\|_{Y}\right]
\end{aligned}
$$

which gives

$$
\begin{aligned}
\|U(t)-\tilde{U}(t)\|_{Y} \leq & e^{w(t-s)}\|U(s)-\tilde{U}(s)\|_{Y}+\int_{s}^{t} e^{w(t-\eta)}\|F(\eta)-\tilde{F}(\eta)\|_{Y} d \eta \\
& +\int_{s}^{t} e^{w(t-\eta)} w\|H(\eta)-\tilde{H}(\eta)\|_{Y} d \eta
\end{aligned}
$$


(II) If we take

$$
\tilde{u}(t) \equiv u(s), \quad \tilde{H}(t) \equiv H(s), \quad \text { for } t \in[0, T]
$$

then the estimate (II) is followed by (I).

(III) By using the fact $\|A x\|_{Y} \leq w\|B x\|_{Y}$ for every $x \in D(B)$ in (II), we obtain

$$
\begin{aligned}
\|A u(t)-A \chi(0)\|_{Y} \leq & w\|B u(t)-B \chi(0)\|_{Y} \\
\leq w[ & \int_{0}^{t} e^{w(t-\eta)}\|F(\eta)-A \chi(0)\|_{Y} d \eta \\
& \left.\quad+\int_{0}^{t} e^{w(t-\eta)} w\|H(\eta)-H(0)\|_{Y} d \eta+\|H(t)-H(0)\|_{Y}\right] .
\end{aligned}
$$

Corollary 2.2. Let $F:[0, T] \rightarrow Y$ be a continuous function and let $u$ be a solution of (2.4). Also, suppose that the assumptions (i)-(v) are satisfied. Then, there exist constants $\alpha=\alpha(T)$ and $\beta=\beta(T)$ such that

$$
\|u(t)-u(s)\|_{X} \leq \alpha\left[\sup _{0 \leq \eta \leq T}\|F(\eta)-A \chi(0)\|_{Y}+\beta\right](t-s), \quad \text { for } 0 \leq s<t \leq T .
$$

Proof. Let us take $b$ as the norm of $B^{-1} \in L(Y, X)$.

Using Proposition 2.1(II) and (III), we obtain

$$
\begin{aligned}
&\|u(t)-u(s)\|_{X} \leq b \|\|B u(t)-B u(s)\|_{Y} \\
& \leq b {\left[\int_{s}^{t} e^{w(t-\eta)}\|F(\eta)-A u(s)\|_{Y} d \eta\right.} \\
& \quad+\int_{s}^{t} e^{w(t-\eta)} w\left\|f\left(\eta, \chi\left(\eta-\tau_{1}\right)\right)-f\left(s, \chi\left(s-\tau_{1}\right)\right)\right\|_{Y} d \eta \\
&\left.+\left\|f\left(t, \chi\left(t-\tau_{1}\right)\right)-f\left(s, \chi\left(s-\tau_{1}\right)\right)\right\|_{Y}\right] \\
& \leq b\left[\sup _{0 \leq \eta \leq T}\|F(\eta)-A \chi(0)\|_{Y}\left(\frac{e^{w t}-e^{w s}}{w}\right)\right.\left.\quad+c\left(e^{w(t-s)}-1\right)\left(\frac{e^{w t}-1}{w}\right)+c\left(\frac{e^{w(t-s)}-1}{w}\right)\right] \\
& \leq b\left[\sup _{0 \leq \eta \leq T}\|F(\eta)-A \chi(0)\|_{Y}+c e^{w T}\right] e^{w T}|t-s| .
\end{aligned}
$$

Set $\alpha=b e^{w T}$ and $\beta=c e^{w T}$. Thus,

$$
\|u(t)-u(s)\|_{X} \leq \alpha\left[\sup _{0 \leq \eta \leq T}\|F(\eta)-A \chi(0)\|_{Y}+\beta\right]|t-s| .
$$


6 Sobolev-type neutral partial differential equations

\section{Existence of solutions}

We start by establishing a result of existence of a solution to the following abstract Cauchy problem:

$$
\begin{gathered}
\frac{d}{d t}\left[B u(t)+f\left(t, \chi\left(t-\tau_{1}\right)\right)\right]+A u(t)=F(t), \quad 0<t \leq \widetilde{T}, \\
u=\chi, \quad \text { on }[-\tau, 0] .
\end{gathered}
$$

Proposition 3.1. If assumptions (i)-(v) are satisfied and $F:[0, \tilde{T}] \rightarrow Y$ is a continuous function, then the Cauchy problem (3.1) has a unique solution.

Proof. Since $A B^{-1}: Y \rightarrow Y$ is a bonded linear operator, it follows that the problem

$$
\begin{gathered}
\frac{d}{d t} w(t)+A B^{-1} w(t)=G(t), \quad t \in(0, \tilde{T}], \\
w(t)=\tilde{\chi}(t), \quad t \in[-\tau, 0]
\end{gathered}
$$

where

$$
G(t)=F(t)+A B^{-1} f\left(t, \chi\left(t-\tau_{1}\right)\right), \quad \forall t \in[0, \tilde{T}]
$$

has a solution $w:[-\tau, \tilde{T}] \rightarrow Y$, given by

$$
w(t)=e^{-t A B^{-1}} \tilde{\chi}(0)+\int_{0}^{t} e^{-(t-s) A B^{-1}} G(s) d s, \quad-\tau \leq t \leq \tilde{T} .
$$

Consequently, a function $u(t)=B^{-1}\left(w(t)-f\left(t, \chi\left(t-\tau_{1}\right)\right)\right)$ is a solution of (3.1). Uniqueness of a solution is obtained by using Proposition 2.1(I).

Our main existence theorem is the following.

Theorem 3.2. Let the assumptions ( $i)-(v)$ be satisfied and suppose that $g$ is a continuous function from $[0, T] \times X \times X$ into $Y$. Then, for any given $\chi(0) \in D(B)$, there exists a solution of the abstract Cauchy problem (2.2) on the subinterval $[-\tau, \tilde{T}] \subset[-\tau, T]$.

Proof. Let $0<\tilde{T} \leq T$ and $R>0$ be real numbers and define the set

$$
S_{0}=S(A \chi(0), R, \widetilde{T}):=\left\{F \in C([0, \widetilde{T}] ; Y): \sup _{0 \leq t \leq \widetilde{T}}\|F(t)-A \chi(0)\|_{Y} \leq R\right\}
$$

It is easy to see that $S_{0}$ is a bounded, closed, and convex subset of $C([0, \tilde{T}] ; Y)$.

Since all the hypotheses of Proposition 3.1 are verified, there exists a solution $u_{F}, F \in$ $S_{0}$, of the problem (3.1).

We define a mapping $Z: S_{0} \rightarrow C([0, \tilde{T}] ; Y)$ by

$$
Z(F)(t):=g\left(t, u_{F}(t), \chi\left(t-\tau_{2}\right)\right)
$$


The proof will be given in three steps.

Step 1. $Z$ is continuous and maps $S_{0}$ into itself.

Let $R>\sup _{0 \leq t \leq T}\|g(t, \chi(0), \chi(0))-A \chi(0)\|_{Y}$ be arbitrary. By the continuity of $g$, we have $\|g(t, x, \xi)-A \chi(0)\|_{Y} \leq R$, for all $t \in[0, T]$, and $x, \xi \in X$ provided $\|(x, \xi)-(\chi(0)$, $\chi(0)) \|_{X} \leq \delta$.

Suppose that $\left\|B^{-1}\right\|_{Y}=b, c>0$ is a constant, and $0<\tilde{T} \leq T$ is such that

$$
b\left[\frac{e^{w \widetilde{T}}-1}{w}\right](R+c) \leq \delta .
$$

In view of these conditions and Proposition 2.1(II), we can easily show that $Z$ maps $S_{0}$ into $S_{0}$. Now, we will show that $Z$ is continuous. To this end, we introduce

$$
V=\left\{u_{F}(t): 0 \leq t \leq \widetilde{T}, F \in S_{0}\right\}
$$

and using Proposition 2.1(II), we observe that $B(V) \subset Y$ is a bounded set. Hence, $V$ is relatively compact in $X$. Therefore, $(t, v) \mapsto g\left(t, v, \chi\left(t-\tau_{2}\right)\right)$ is uniformly continuous on $[0, \tilde{T}] \times V$ and Proposition 2.1(I) implies that $Z: S_{0} \rightarrow C([0, \tilde{T}] ; Y)$ is continuous.

Step 2. $Z\left(S_{0}\right)$ is equicontinuous.

Let $F \in S_{0}$ and $t_{1}, t_{2} \in[0, \tilde{T}]$. Then, if $0<t_{1}<t_{2} \leq \tilde{T}$,

$$
\left\|Z(F)\left(t_{1}\right)-Z(F)\left(t_{2}\right)\right\|_{Y}=\left\|g\left(t_{1}, u_{F}\left(t_{1}\right), \chi\left(t_{1}-\tau_{2}\right)\right)-g\left(t_{2}, u_{F}\left(t_{2}\right), \chi\left(t_{2}-\tau_{2}\right)\right)\right\|_{Y} .
$$

The right-hand side tends to zero as $t_{2}-t_{1} \rightarrow 0$, since from Corollary 2.2 we obtain $\left\|u_{F}\left(t_{1}\right)-u_{F}\left(t_{2}\right)\right\|_{X} \leq \alpha(R+\beta)\left|t_{1}-t_{2}\right|$ and $g$ is a uniformly continuous function. Thus, $Z$ maps $S_{0}$ into an equicontinuous family of functions.

Step 3. $Z$ maps $S_{0}$ into precompact set in $Y$.

Let $t \in[0, \tilde{T}]$ be fixed. Then, image of $\{t\} \times V \times\{\chi\}$ under continuous function $g$ is precompact in $Y$. Therefore, the set $\left\{Z(F)(t): F \in S_{0}\right\}$ is precompact in $Y$.

As a consequence of Step 2 and Step 3 together with the Ascoli-Arzela theorem, we infer that $Z\left(S_{0}\right)$ is relatively compact in $Y$. Hence, by Schauder's fixed point theorem, we deduce that the operator $Z$ has a fixed point. This means that the problem (2.2) has a solution.

Next we will prove the following global existence result.

Theorem 3.3. Suppose that all the hypotheses of Theorem 3.2 are satisfied. Then, (2.2) has a solution either on $[-\tau, T]$ or on the maximal interval of existence $\left[-\tau, t_{\max }\right), 0<t_{\max } \leq T$, and in the latter case, $\lim _{t \rightarrow t_{\max }-}\|u(t)\|_{X}=\infty$.

Proof. Since all the assumptions of Theorem 3.2 are satisfied, there exists a solution $u$ of (1.1) on $[-\tau, \tilde{T}]$. 
Suppose $\tilde{T}<T$ and consider the problem

$$
\begin{gathered}
\frac{d}{d t}\left[B w(t)+f\left(t+\tilde{T}, \tilde{\chi}\left(t-\tau_{1}\right)\right)\right]+A w(t)=g\left(t+\tilde{T}, \tilde{\chi}\left(t-\tau_{2}\right), w(t)\right), \quad 0<t \leq T-\tilde{T}, \\
w=\tilde{\chi}, \quad \text { on }[-\tau-\widetilde{T}, 0],
\end{gathered}
$$

where

$$
\tilde{\chi}(t)= \begin{cases}\chi(t+\tilde{T}), & t \in[-\tau-\tilde{T},-\tilde{T}] \\ u(t+\tilde{T}), & t \in[-\widetilde{T}, 0] .\end{cases}
$$

Since $\tilde{\chi}(0)=u(\tilde{T}) \in D(B), f$ satisfies the assumption (v), and $g$ is a continuous function, we may proceed as before and prove the existence of a solution $w:\left[-\tau, T_{1}\right] \rightarrow X, 0<T_{1} \leq$ $T-\widetilde{T}$, of the considered problem (3.10).

Then, the function $\bar{u}:\left[-\tau, \widetilde{T}+T_{1}\right] \rightarrow X$, given by

$$
\bar{u}(t)= \begin{cases}u(t), & t \in[-\tau, \tilde{T}], \\ w\left(t-T_{0}\right), & t \in\left[\widetilde{T}, \widetilde{T}+T_{1}\right]\end{cases}
$$

is a solution of $(2.2)$ on $\left[-\tau, \widetilde{T}+T_{1}\right]$. Continuing this way, we may prove the existence either on the whole interval $[-\tau, T]$ or on the maximal interval of existence $\left[-\tau, t_{\max }\right)$, $0<t_{\max } \leq T$. In the latter case, if $\lim _{t \rightarrow t_{\max }-}\|u(t)\|_{X}<\infty$, then as $u(t) \in D(B)$ for $t \in$ $\left[0, t_{\max }\right)$, we have that $\lim _{t \rightarrow t_{\max }}-u(t)$ is in the closure of $D(B)$ in $X$, and if it in $D(B)$, then proceeding as before, we may extend $u(t)$ beyond $t_{\max }$, but this will contradict the definition of the maximal interval of existence. Therefore, $\lim _{t \rightarrow t_{\max }}\|u(t)\|_{X}=\infty$.

\section{An example}

In this section, we consider an example to illustrate the abstract results.

Consider the following initial boundary value problem:

$$
\begin{gathered}
\frac{\partial}{\partial t}\left[(1-\Delta) w(t, x)+\int_{\Omega} k_{1}(t, x, y) b_{1}\left(w\left(t-\tau_{1}, y\right)\right) d y\right]-\Delta w(t, x) \\
=\int_{\Omega} k_{2}(t, x, y) b_{2}\left(w(t, y), w\left(t-\tau_{2}, y\right)\right) d y, \quad x \in \Omega, t \in(0, T] \\
w(t, x)=0, \quad t \geq 0, x \in \partial \Omega \\
h_{0}(w(t, x))=\phi_{0}(x), \quad x \in \bar{\Omega}, t \in[-\tau, 0]
\end{gathered}
$$

where $\Omega$ is a bounded domain in $\mathbb{R}^{n}$ with sufficiently smooth boundary $\partial \Omega$, and $\Delta$ is the $N$-dimensional Laplacian. To represent problem (4.1) as the Cauchy problem (1.1), we take $X=L^{p}(\Omega), Y=C(\bar{\Omega})$, and define $u(t)(x)=w(t, x), B_{1}\left(u\left(t-\tau_{1}\right)\right)(x)=b_{1}(w(t-$ $\left.\left.\tau_{1}, x\right)\right)$, and $B_{2}\left(u(t), u\left(t-\tau_{2}\right)\right)(x)=b_{2}\left(w(t, x), w\left(t-\tau_{1}, x\right)\right)$. The operators $A$ and $B$ are 
defined by

$$
\begin{gathered}
A z=-\Delta z, \quad \forall z \in D(A), \\
B \tilde{z}=\tilde{z}-\Delta \widetilde{z}, \quad \forall \tilde{z} \in D(B),
\end{gathered}
$$

with the domains

$$
D(A)=D(B)=W_{0}^{1, p}(\Omega) \cap W^{2, p}(\Omega), \quad p>n .
$$

Then, $A$ and $B$ are closed, linear operators. Furthermore, $A$ is an accerative operator and $D(A)$ is compactly imbedded in $Y$. Therefore, $A$ and $B$ verify the assumptions (i)-(iii).

We assume the following conditions.

(i) $k_{1}(t, x, y)$ and $k_{2}(t, x, y)$ are real-valued Lipschitz continuous and continuous functions, respectively, on $[0, T] \times \Omega \times \Omega$ satisfying

$$
\sup _{x \in \Omega} \int_{\Omega}\left(\int_{\Omega}\left|k_{i}(t, x, y)\right|^{q} d y\right)^{1 / q} d x<\infty, \quad \frac{1}{p}+\frac{1}{q}=1, i=1,2 .
$$

(ii) $b_{1}$ is a real-valued Lipschitz continuous function and $b_{2}$ is a real-valued continuous function.

Define $f:[0, T] \times L^{p}(\Omega) \rightarrow C(\bar{\Omega})$ and $g:[0, T] \times L^{p}(\Omega) \times L^{p}(\Omega) \rightarrow C(\bar{\Omega})$ by

$$
\begin{gathered}
f(t, \xi)(x)=\int_{\Omega} k_{1}(t, x, y) B_{1}(\xi)(y) d y, \\
g(t, v, \eta)(x)=\int_{\Omega} k_{2}(t, x, y) B_{2}(v, \eta)(y) d y,
\end{gathered}
$$

respectively. Clearly, $f$ is a Lipschitz continuous function and $g$ is a continuous function. For nonlocal history function $h_{0}$, we may have any of the following.

(I) $h_{0}(\psi)(x)=\int_{-\tau}^{0} k(s) \psi(s)(x) d s$, for $x \in \Omega$ and $\psi \in \mathscr{C}_{0}$, where $k \in L^{1}(-\tau, 0)$ with $\kappa:=\int_{-\tau}^{0} k(s) d s \neq 0$.

(II) $h_{0}(\psi)(x)=\sum_{i=1}^{n} c_{i} \psi\left(\theta_{i}\right)(x)$ for $x \in \Omega$ and $\psi \in \mathscr{C}_{0}$, where $-\tau \leq \theta_{1} \leq \theta_{2}<\cdots<$ $\theta_{n} \leq 0, n \in \mathbb{N}, c_{i} \geq 0$, and $C:=\sum_{i=1}^{n} c_{i} \neq 0$.

(III) $h_{0}(\psi)(x)=\sum_{i=1}^{n}\left(c_{i} / \epsilon_{i}\right) \int_{\theta_{i}-\epsilon_{i}}^{\theta_{i}} \psi(s)(x) d s$ for $x \in \Omega$ and $\psi \in \mathscr{C}_{0}$, where $\theta_{i}$ and $c_{i}$ are as in (II) and $\epsilon_{i}>0$, for $i=1,2, \ldots, n$.

Define $h(\psi)(t)=h_{0}(\psi)$, for $t \in[-\tau, 0]$. Let $\phi(t) \equiv \phi_{0}$ on $[-\tau, 0], \phi_{0} \in D(B)$. For (I), we may take $\chi(t)=(1 / \kappa) \phi_{0}$, and for (II) as well as for (III), we may take $\chi(t)=(1 / C) \phi_{0}$ on $[-\tau, 0]$. Then, $h$ satisfies (iv). Therefore, all the assumptions of Theorem 3.2 are satisfied and hence, the problem (4.1) admits a solution.

\section{References}

[1] D. Bahuguna, Existence, uniqueness and regularity of solutions to semilinear nonlocal functional differential problems, Nonlinear Analysis. Theory, Methods \& Applications. An International Multidisciplinary Journal. Series A: Theory and Methods 57 (2004), no. 7-8, 1021-1028.

[2] K. Balachandran and M. Chandrasekaran, Existence of solutions of a delay differential equation with nonlocal condition, Indian Journal of Pure and Applied Mathematics 27 (1996), no. 5, 443449. 
[3] K. Balachandran and R. Sakthivel, Existence of solutions of neutral functional integrodifferential equation in Banach spaces, Indian Academy of Sciences. Proceedings. Mathematical Sciences 109 (1999), no. 3, 325-332.

[4] K. Balachandran and K. Uchiyama, Existence of solutions of nonlinear integrodifferential equations of Sobolev type with nonlocal condition in Banach spaces, Indian Academy of Sciences. Proceedings. Mathematical Sciences 110 (2000), no. 2, 225-232.

[5] H. Brill, A semilinear Sobolev evolution equation in a Banach space, Journal of Differential Equations 24 (1977), no. 3, 412-425.

[6] L. Byszewski, Theorems about the existence and uniqueness of solutions of a semilinear evolution nonlocal Cauchy problem, Journal of Mathematical Analysis and Applications 162 (1991), no. 2, 494-505.

[7] L. Byszewski and H. Akca, Existence of solutions of a semilinear functional-differential evolution nonlocal problem, Nonlinear Analysis. Theory, Methods \& Applications. An International Multidisciplinary Journal. Series A: Theory and Methods 34 (1998), no. 1, 65-72.

[8] L. Byszewski and V. Lakshmikantham, Theorem about the existence and uniqueness of a solution of a nonlocal abstract Cauchy problem in a Banach space, Applicable Analysis. An International Journal 40 (1991), no. 1, 11-19.

[9] X. Fu and K. Ezzinbi, Existence of solutions for neutral functional differential evolution equations with nonlocal conditions, Nonlinear Analysis. Theory, Methods \& Applications. An International Multidisciplinary Journal. Series A: Theory and Methods 54 (2003), no. 2, 215-227.

[10] M. E. Hernández, Existence results for partial neutral functional differential equations with nonlocal conditions, Cadernos De Matemática 2 (2001), no. 2, 239-250.

[11] M. E. Hernández and H. R. Henríquez, Existence results for partial neutral functional-differential equations with unbounded delay, Journal of Mathematical Analysis and Applications 221 (1998), no. 2, 452-475.

[12] T. Kato, Nonlinear semigroups and evolution equations, Journal of the Mathematical Society of Japan 19 (1967), 508-520.

[13] A. Pazy, Semigroups of Linear Operators and Applications to Partial Differential Equations, Applied Mathematical Sciences, vol. 44, Springer, New York, 1983.

[14] R. E. Showalter, Existence and representation theorems for a semilinear Sobolev equation in Banach space, SIAM Journal on Mathematical Analysis 3 (1972), 527-543.

[15] A nonlinear parabolic-Sobolev equation, Journal of Mathematical Analysis and Applications 50 (1975), 183-190.

Shruti Agarwal: Department of Mathematics, Indian Institute of Technology, Kanpur - 208 016, India

Dhirendra Bahuguna: Department of Mathematics, Indian Institute of Technology,

Kanpur - 208 016, India

E-mail address: d_bahuguna@yahoo.com 


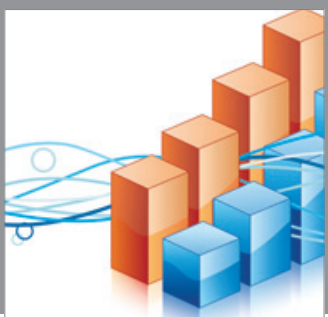

Advances in

Operations Research

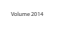

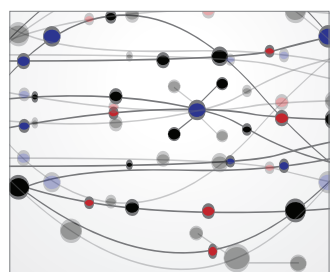

\section{The Scientific} World Journal
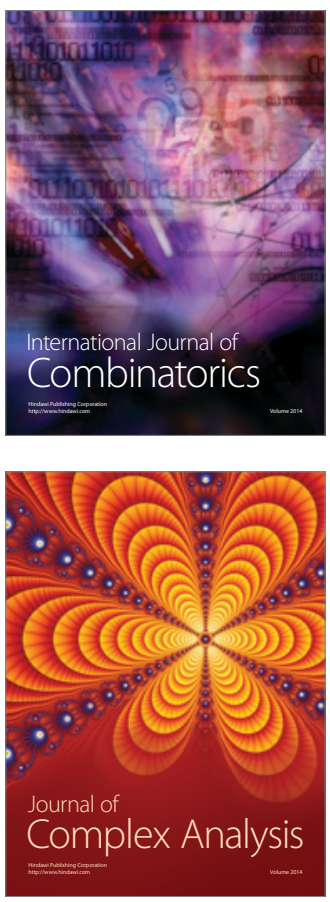

International Journal of

Mathematics and

Mathematical

Sciences
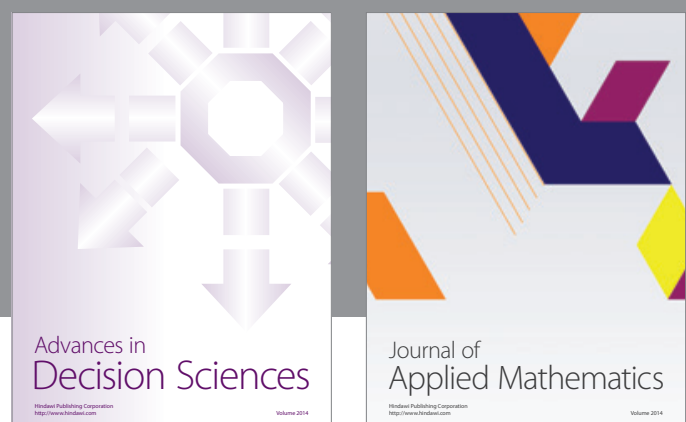

Journal of

Applied Mathematics
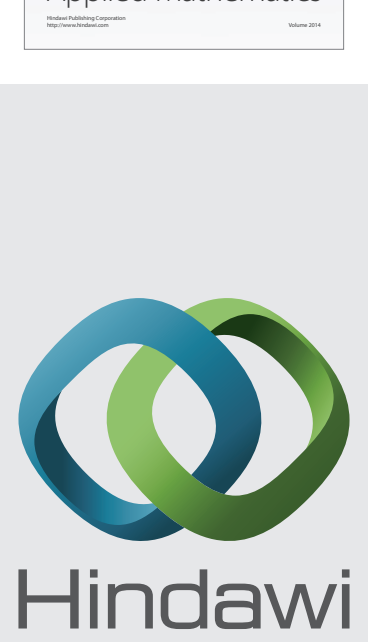

Submit your manuscripts at http://www.hindawi.com
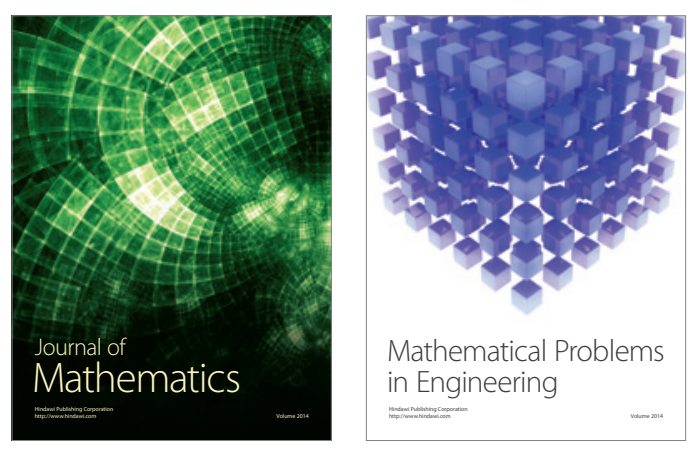

Mathematical Problems in Engineering
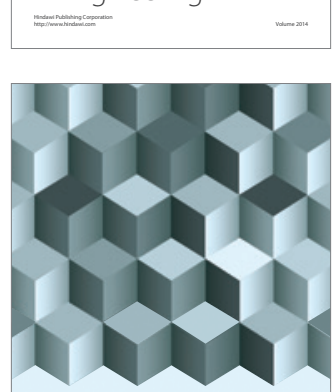

Journal of

Function Spaces
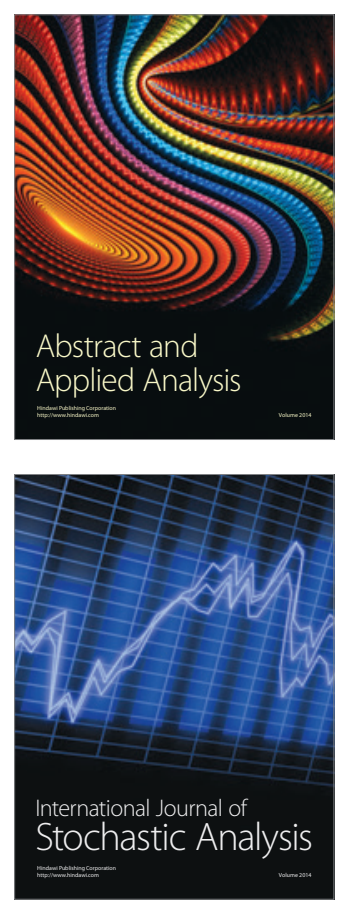

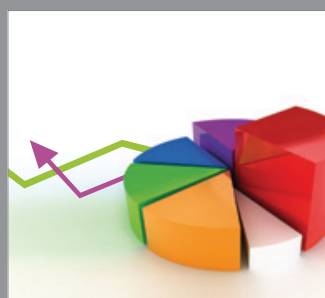

ournal of

Probability and Statistics

Promensencen
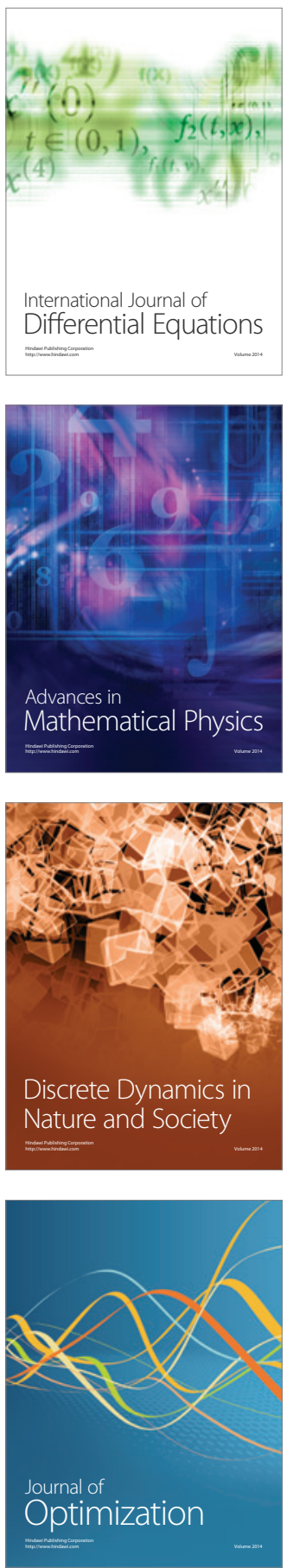